\title{
Suggested statistical reappraisal of data from comparative study of febuxostat and allopurinol in chronic kidney disease
}

\begin{abstract}
Alan Zelicoff
Received: 19 September 2014 / Accepted: 21 September 2014 / Published online: 3 October 2014

(C) International League of Associations for Rheumatology (ILAR) 2014
\end{abstract}

To the Editor:

Tsuruta et al. [1] have called attention to the potential value of febuxostat in the management of hyperuricemia in patients with chronic kidney disease (CKD). Their important contribution augments existing literature regarding the safety and efficacy of febuxostat in this population [2] as we await results from prospective studies on the utility of the drug in slowing the progression of CKD in hyperuricemic individuals without gout [3].

In their retrospective study, Tsuruta et al. conclude that uric acid levels are lower and glomerular filtration rate are better preserved among patients taking febuxostat as compared to allopurinol (Tables 3 and 4 of [1]). However, their statistical analysis is based on a repeated Student's $t$ test but without adjustment for associated multiple comparisons via Bonferroni, Sidak-Holm, or related corrections [4]. Might the authors apprise interested readers of the relevant corrected statistical values?

More compelling still would be an analysis with a randomeffects model or perhaps a survival analysis (perhaps utilizing percent of patients maintaining some level of glomerular filtration rate (GFR)), as these approaches would fully take into the time-series nature of their data.

Disclosures None

\section{References}

1. Tsuruta Y, Mochizuki T, Moriyama T, Itabashi M, Takei T, Tsuchiya $\mathrm{K}$, et al (2014) Switching from allopurinol to febuxostat for the treatment of hyperuricemia and renal function in patients with chronic kidney disease. Clin Rheumatol. doi:10.1007/s10067-014-2745-5

2. Shibagaki Y, Ohno I, Hosoya T, Kimura K (2014) Safety, efficacy and renal effect of febuxostat in patients with moderate-to-severe kidney dysfunction. Hypertens Res Off J Jpn Soc Hypertens. doi:10.1186/ 1745-6215-15-26

3. Hosoya T, Kimura K, Itoh S, Inaba M, Uchida S, Tomino Y et al (2014) The effect of febuxostat to prevent a further reduction in renal function of patients with hyperuricemia who have never had gout and are complicated by chronic kidney disease stage 3 : study protocol for a multicenter randomized controlled study. Trials 15:26

4. Glantz SA (2012) Primer of Biostatistics. McGraw-Hill, New York
A. Zelicoff $(\bowtie)$

Environmental and Occupational Health and Epidemiology, College for Public Health and Social Justice, St. Louis University, 3545 Lafayette Ave., Room 466, Salus Center, St. Louis, MO 63104, USA e-mail: zelicoff@slu.edu 